\title{
Análise de sensibilidade do consumo de gasolina C entre julho de 2001 e dezembro de 2008: \\ Política Tributária Estadual como instrumento de \\ políticas energéticas e ambientais
}

Thaís Machado de M. Vilela

Mestre em Economia pelo Instituto de Economia da UFRJ

Helder Queiroz Pinto Junior

Professor do Instituto de Economia da UFRJ

\section{Palauras-chave \\ gasolina C, elasticidade-preço da demanda, ICMS.}

\section{Classificação JEL C32, C51 H39.}

\section{Key words}

gasoline, demand

price-elasticity, ICMS

JEL Classification C32, C51 H39.

\section{Resumo}

A partir de uma análise descritiva dos dados de consumo de gasolina C por estado é possível notar diferentes comportamentos entre os mesmos. Desta forma, a análise de sensibilidade da demanda por gasolina $\mathrm{C}$ a variações no preço no Brasil deve considerar essa multiplicidade de respostas. Através de uma abordagem econométrica tradicional de séries de tempo, procura-se, neste artigo, avaliar as diferentes reações a mudanças no preço, mais especificamente, a mudanças no imposto estadual ICMS sobre a gasolina C. O cálculo da elasticidade-imposto permite verificar os efeitos da adoção de políticas públicas estaduais nos planos ambiental e energético.

\section{Abstract}

This paper is focused on a descriptive analysis of gasoline consumption to identify different consumers' behaviors among Brazilian States. The sensitive analysis of gasoline demand to price variation must consider a larger set of variables. Trough a traditional econometric time series approach, this paper aims at assessing the different reactions to price changes, more specifically, changes in state tax, ICMS, over gasoline. The tax-elasticity calculation allows us to verify the possible use of state public policies, specially concerning environmental and energy issues. 


\section{1_ Introdução}

No Brasil, o tema da evolução do consumo de gasolina $\mathrm{C}$, definida como uma combinação de gasolina pura com álcool etílico anidro carburante, insere-se num contexto bastante amplo, envolvendo as políticas energética, ambiental e tributária. Com relação às duas primeiras, temse que uma das finalidades dessas políticas é a de maximizar o bem-estar da sociedade, e, para isso, a formulação de ambas as políticas deve considerar as diversas esferas de ação, assim como os impactos nas diferentes áreas, como, por exemplo, nas áreas econômica, tecnológica, ambiental, política e social.

A mensuração da elasticidade-preço da demanda da gasolina tem se revelado um desafio para os analistas, em particular, graças ao advento dos carros flex, que permitem aos consumidores arbitrarem entre o álcool e a gasolina C, em função dos preços relativos desses combustíveis. À dificuldade para estimar a elasticidades associa-se igualmente $\mathrm{O}$ problema da incidência de tributos sobre os consumidores.

No Brasil, no caso específico da gasolina $\mathrm{C}$, objeto de estudo deste trabalho, existem três impostos federais, a CIDE, o PIS/PASEP e o COFINS, e um imposto estadual, o ICMS. O primeiro desafio apresentado, portanto, é definir qual imposto deve ser estudado. Para tal, é preciso analisar a existência de diferentes comportamentos quanto à demanda por gasolina nos Estados brasileiros. Sendo assim, a ideia é verificar a necessidade de regionalizar a política pública, a fim de captar melhor a diversidade, caso exista, de consumo de gasolina C entre os Estados. Para a implementação dessas políticas, há um conjunto complexo de instrumentos, sendo os impostos uma das ferramentas governamentais possíveis de ser utilizadas.

Desta forma, o passo seguinte seria o de calcular as respostas dos consumidores às mudanças nesse imposto e, consequentemente, verificar a viabilidade de se utilizar a política tributária estadual como instrumento de políticas energéticas e ambientais.

Assim, este artigo está estruturado da seguinte maneira. Primeiro, a evolução do mercado de gasolina $\mathrm{C}$ no Brasil é analisada a partir da década de 1970, considerando, nesta seção, as diversidades estaduais, assim como a estruturação do preço da gasolina C. Em seguida, na seção 3, a estimação da elasticidade-imposto da demanda é realizada utilizando-se a abordagem econométrica tradi- 
cional de séries temporais, considerando o período de julho de 2001 a dezembro de 2006. Por fim, as considerações finais são elaboradas.

\section{2_Evolução do mercado de gasolina C no Brasil}

A partir da década de 1970, o mercado de gasolina $\mathrm{C}$ sofreu diversas alterações. Vejamos no Gráfico 2.1.

Em 1973, os preços do petróleo no mercado internacional quadruplicaram em apenas três meses. O impacto do aumento dos preços do petróleo na inflação preocupava o governo, que repassou o aumento apenas para os preços da gasolina, subsidiando, assim, o consumo do diesel. Como resultado dessa medida, houve a "dieselização" da frota nacional.

Contudo, cabe lembrar que, assim como a gasolina, o diesel é também um combustível derivado de petróleo, cujo preço havia quadruplicado em apenas três meses. Ao contrário dos demais países, porém, o governo brasileiro optou por evitar a recessão econômica, e,

\section{Gráfico 2.1_Consumo anual de combustíveis automotivos (milhões de tep)}

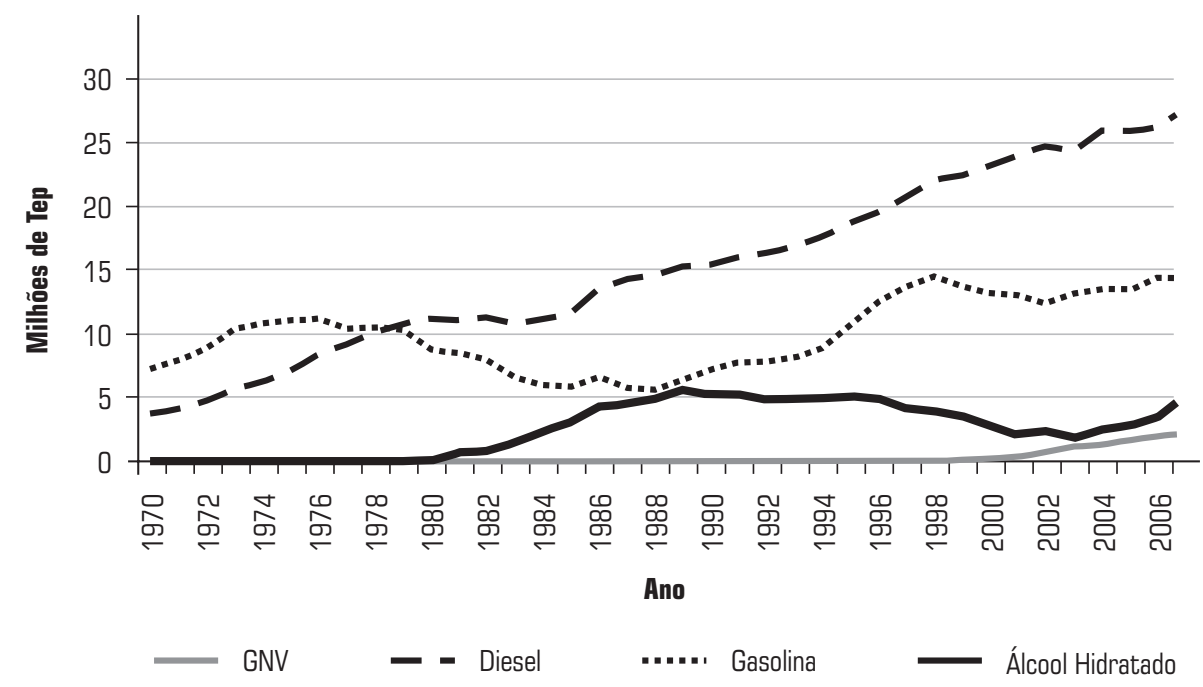

Fonte: BEN (2008) 
com o objetivo de reduzir ainda mais a dependência e vulnerabilidade brasileira aos preços internacionais do petróleo, o governo federal instituiu, em 14 de novembro de 1975, o Programa Nacional do Álcool.

Inicialmente, o programa visava à adição de álcool anidro à gasolina; todavia, com o "segundo choque do petróleo" e com o novo patamar de US\$30 o barril do petróleo em 1980, os gastos com as importações de petróleo e de seus derivados aumentaram consideravelmente. Diante dessa situação, o governo passou a incentivar, através de políticas fiscais e tributárias, o uso do álcool etílico hidratado carburante como combustível substituto à gasolina. Em 19 de setembro de 1979, o governo e a Anfavea assinaram um protocolo de compromisso com o objetivo de desenvolver e comercializar veículos a álcool no Brasil.

A adição de mais de 10\% de álcool aos motores convencionais da época gerou preocupações, por exemplo, quanto à compatibilidade de material metálico do veículo e a possibilidade de corrosão. Dadas essas limitações, foi necessário modificar os veículos para que pudessem receber álcool hidratado como combustível. Tais modificações possibilitaram o avanço da tecnologia automoti- va relacionada ao etanol e garantiram ao Brasil papel de destaque no mundo.

Contudo, no final da década de 1980, os preços do petróleo no mercado internacional reduziram-se à metade, de US $\$ 30$ para US\$ 15 o barril, período denominado "contrachoque do petróleo". Nesse cenário, tornou-se custoso para o governo financiar a produção de álcool carburante, especialmente de álcool hidratado. Logo, os subsídios foram eliminados. Além disso, o crescimento desarticulado da frota a álcool, a redução dos estoques de segurança de dois meses para nove dias, a queda da produção de cana nas últimas safras à ocasião em razão das práticas de preços comprimidos para a cana colhida, o aumento das exportações de açúcar graças à alta dos preços internacionais desse, entre outros fatores, contribuíram para o estabelecimento de uma crise de abastecimento. Dentro desse contexto, a maior parte dos consumidores voltou a utilizar a gasolina como combustível. Mas o prejuízo dos consumidores, assim como a desconfiança em relação à garantia de oferta de álcool hidratado, resultaram na descrença quanto ao uso do álcool como combustível substituto.

Já, na década de 1990, o governo federal incentivou o uso do gás natural 
como combustível substituto da gasolina. A descoberta de reservas na Bacia de Campos (RJ) e o avanço das negociações, a partir de 1994, para a importação de gás boliviano aumentaram a disponibilidade de gás natural no mercado brasileiro. Com isso, além do apoio governamental e o preço baixo do produto, por causa do aumento da oferta, muitos consumidores fizeram a conversão necessária em seu veículo para usarem gás natural.

Em março de 2003, os carros com tecnologia flex-fuel foram introduzidos no mercado de automóveis no Brasil. A tecnologia flex-fuel consiste na possibilidade de utilizar gasolina, álcool ou qualquer proporção desses combustíveis num mesmo tanque. $\mathrm{O}$ consumo, entretanto, por quilômetro percorrido, difere entre esses. Motores movidos a gasolina percorrem dez quilômetros por litro, enquanto os abastecidos com álcool percorrem apenas sete, ou seja, o álcool possui autonomia 30\% menor do que a gasolina, de forma que a adoção do álcool como combustível só se justifica economicamente se seu preço for no máximo igual a 0,7 do preço da gasolina. Caso contrário, a gasolina é a opção mais vantajosa. O fato é que essa nova tecnologia possibilitou a recuperação das vendas de álcool hidratado até então em tendência decrescente (Gráfico 2.1).

Cabe mencionar que, atualmente, existem também os carros "tricombustíveis", que permitem ao consumidor escolher não só entre a gasolina e o álcool, mas também entre o gás natural veicular (GNV). Segundo o Instituto Brasileiro de Petróleo, Gás e Biocombustíveis, para a gasolina ser mais econômica que o GNV, seu preço deve ser $20 \%$ menor que o do gás. Já, no caso do álcool, esse deverá ser $50 \%$ menor que o preço do GNV. Na prática, o preço do gás natural veicular é menor que o preço de ambos os combustíveis, de forma que é sempre mais vantajoso abastecer com gás natural. Os maiores consumidores de GNV são os taxistas, uma vez que são grandes as distâncias percorridas, justificando o investimento inicial no kit gás. É possível considerar o mercado de gás como um mercado próprio, independentemente das oscilações dos mercados de gasolina e álcool hidratado.

\section{1_ Diferenças estaduais}

\subsection{1_ Consumo de gasolina C}

Como não há uma medida quantitativa exata do consumo de gasolina $\mathrm{C}$, as vendas desse combustível pelas distribuidoras são utilizadas como medida de demanda. 
De acordo com o Gráfico 2.2, é possível notar que os Estados das regiões Sul e Sudeste são os maiores consumidores de gasolina $\mathrm{C}$ no Brasil. De fato, o consumo nessas duas regiões corresponde a $68,50 \%$ do consumo total desse combustível. São Paulo é o Estado que lidera essa estatística, com 27,86\%. Tal fato está bastante relacionado com o tamanho da frota de automóveis. Ao analisar os dados referentes à frota de automóveis disponibilizados pelo Dena- tran, as regiões Sul e Sudeste possuem a maior concentração de automóveis. Em dezembro de 2007, essa participação correspondia a, aproximadamente, $81 \%$ da frota de automóveis nacionais.

Dentro desse contexto, é possível, então, identificar diferenças quanto ao consumo de gasolina $\mathrm{C}$ entre os Estados. Essas diferenças são resultado de diversos fatores, entre esses, o tamanho populacional, a malha rodoviária, o desenvolvimento econômico e os hábitos

\section{Gráfico 2.2_ Venda de gasolina C pelas distribuidoras, em 2008}

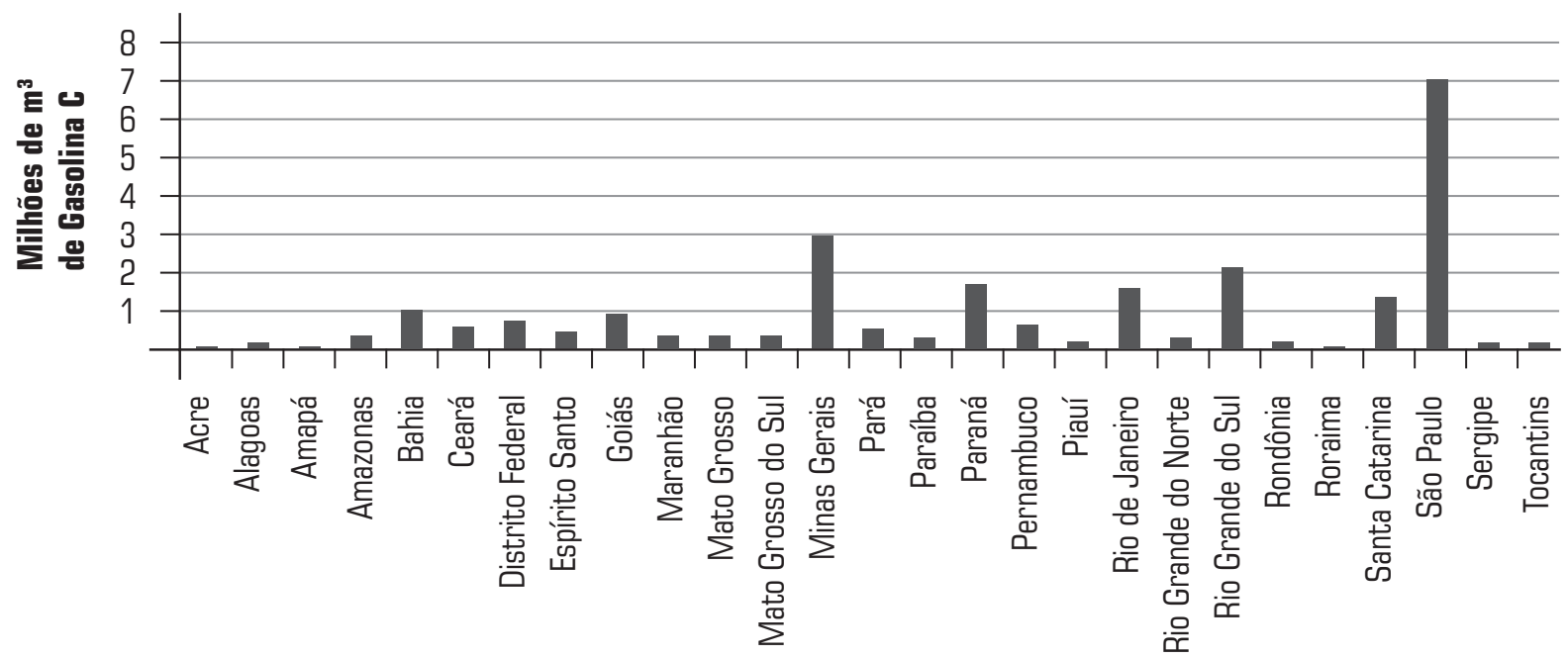

Unidades da Federação

Fonte: ANP (2009). 
dos consumidores. Quanto ao último fator, apesar de extremamente importante, não pode ainda ser mensurado e, por isso, é considerado apenas um resíduo na explicação da variação do consumo de gasolina C.

Além desses fatores, a assimetria identificada em relação ao comportamento da demanda por gasolina $\mathrm{C}$ nos Estados brasileiros pode ser consequência das diferentes reações dos Estados a mudanças no preço desse combustível, sendo esse o fator de interesse deste trabalho.

\subsection{2_Preço da gasolina C}

Dado que a gasolina C é formada pela combinação entre a gasolina pura, ou A, e o álcool anidro, é preciso considerar a composição dos custos da gasolina A desde o produtor e do álcool anidro a fim de entender os componentes do preço da gasolina C.

Considerando primeiro o custo da gasolina pura, os fatores que incidem sobre esse são:

i. o preço de realização dos produtores;

ii. os impostos federais PIS/PASEP, COFINS e CIDE;

iii. o imposto estadual ICMS;

iv. a substituição tributária referente ao ICMS; v. o frete relativo ao transporte da gasolina $\mathrm{A}$ até a base de distribuição. Ao mesmo tempo, os fatores que incidem sobre o custo do álcool anidro são: a) o preço do álcool anidro; b) o frete de coleta.

Desta forma, para a composição final do custo da gasolina $\mathrm{C}$ para os postos revendedores, é preciso acrescentar a margem de distribuição das distribuidoras, assim como o frete da base de distribuição até os postos. Sendo assim, o preço final da gasolina $\mathrm{C}$ na bomba é a soma do preço de aquisição mais a margem de revenda.

Como o objetivo deste trabalho é o de avaliar os impactos de mudanças no ICMS sobre o consumo de gasolina C, será detalhado, em seguida, apenas o ICMS.

\subsubsection{1_ ICMS sobre a gasolina C}

O imposto sobre operações relativas à Circulação de Mercadorias e sobre Prestação de Serviços de Transporte Interestadual e Intermunicipal e de Comunicação (ICMS), único imposto brasileiro sobre valor agregado, passou a incidir sobre a gasolina C em 1989. A Constituição Federal de 1988 atribuiu aos Estados brasileiros a competência de tributar as operações de comercialização de gasolina C. 
Por ser um imposto estadual, as alíquotas referentes ao ICMS podem ser diferentes entre os Estados (Tabela 2.1). Além disso, o ICMS está associado ao consumo e não à renda, uma vez que é um imposto indireto. Por fim, por ser um imposto não cumulativo, a incidência dele é somente sobre o valor acrescido em cada etapa de produção. Portanto, o montante de imposto cobrado nas operações anteriores é abatido em cada nova etapa.

Os fatores geradores para a incidência do ICMS são três:

i. a circulação de mercadorias dentro de um mesmo Estado ou entre Estados;

ii. a prestação de serviço de comunicação;

iii. a prestação de serviços de transporte intermunicipal e interestadual.

Para o cálculo do imposto estadual, o preço de venda considerando o próprio imposto, modalidade de cálculo denominada "por dentro", é utilizado. Contudo, a fim de reduzir a sonegação fiscal, foi estabelecido o regime de substituição tributária.

De acordo com essa modalidade de tributação, um contribuinte é determinado como responsável pelo recolhimento do imposto devido nas demais

\section{Tabela 2.1_Alíquota do ICMS sobre a gasolina em janeiro de 2008}

\begin{tabular}{|c|c|}
\hline Unidades da Federação & Alíquota (\%) \\
\hline Acre & 25 \\
\hline Alagoas & 27 \\
\hline Amazonas & 25 \\
\hline Amapá & 25 \\
\hline Bahia & 27 \\
\hline Ceará & 27 \\
\hline Distrito Federal & 25 \\
\hline Espírito Santo & 27 \\
\hline Goiás & 26 \\
\hline Maranhão & 27 \\
\hline Minas Gerais & 25 \\
\hline Mato Grosso do Sul & 25 \\
\hline Mato Grosso & 25 \\
\hline Pará & 30 \\
\hline Paraíba & 27 \\
\hline Pernambuco & 27 \\
\hline Piauí & 25 \\
\hline Paraná & 26 \\
\hline Rio de Janeiro & 31 \\
\hline Rio Grande do Norte & 25 \\
\hline Rondônia & 25 \\
\hline Roraima & 25 \\
\hline Rio Grande do Sul & 25 \\
\hline Santa Catarina & 25 \\
\hline Sergipe & 27 \\
\hline São Paulo & 25 \\
\hline Tocantins & 25 \\
\hline
\end{tabular}

Fonte: Sindicom (2009). 
operações. No que se refere especificamente à gasolina, as refinarias são as responsáveis por recolher o ICMS devido pelos contribuintes referentes às operações seguintes. Nesse caso, a base de cálculo é obtida pelo somatório do valor da operação realizada pelo substituto tributário, ou seja, as refinarias, mais o montante dos valores de seguro, fretes e de outros encargos cobrados ou transferíveis para as distribuidoras e postos revendedores e, finalmente, a margem de valor agregado, inclusive o lucro, relativa às operações posteriores.

Vale mencionar que a margem considerada para o cálculo do imposto é estabelecida com base em preços usualmente praticados no mercado, obtida por levantamento ou através de informações com os agentes desse mercado.

Além disso, as alíquotas do ICMS são classificadas em internas, quando a comercialização ocorre dentro de um mesmo Estado, ou, caso contrário, interestaduais. As alíquotas internas referentes à gasolina $\mathrm{C}$ pouco mudaram nos últimos oito anos, não havendo, a princípio, razão para considerar o ICMS tema de pesquisa. Contudo, a possibilidade de utilizar o imposto estadual como instrumento de política energética e política ambiental justifica o interesse.
Por fim, visto que o objeto deste estudo é a gasolina C, é preciso analisar a incidência do imposto sobre o álcool anidro. Em relação às operações de aquisição de álcool anidro pelas distribuidoras às destilarias e usinas, não há ICMS. O imposto estadual sobre o álcool anidro é recolhido junto com o ICMS sobre a gasolina $\mathrm{A}$.

\section{3_ Elasticidade-imposto da demanda por gasolina C no Brasil}

Como mencionado anteriormente, o objetivo deste trabalho é o de avaliar o impacto de mudanças no imposto estadual ICMS sobre o consumo de gasolina $\mathrm{C}$ no Brasil, mais especificamente, nos Estados brasileiros. Entretanto, por uma questão de disponibilidade de dados, apenas sete Estados são considerados, quais sejam: Ceará, Pernambuco, Sergipe, Rio de Janeiro, Paraná, Rio Grande do Sul e Mato Grosso do Sul.

\subsection{Hipóteses do modelo}

De acordo com a legislação brasileira, são os produtores, a refinaria, a distribuidora e os revendedores que pagam o ICMS sobre a gasolina C. Contudo, isso não significa que a incidência desse imposto recaia de fato sobre os produto- 
res. A incidência tributária depende de alguns fatores, sendo a estrutura de mercado o mais importante deles.

No caso específico do mercado revendedor de gasolina $\mathrm{C}$, esse é considerado competitivo. Contudo, é preciso estar ciente de que tal mercado apresenta características propícias à formação de cartéis. No entanto, apesar de possíveis focos de cartelização no mercado revendedor, esses não constituem a real estrutura de mercado, sendo, portanto, apenas casos isolados. Desta forma, o mercado de gasolina $\mathrm{C}$ pode ser considerado competitivo. Posto isso, as curvas de oferta e de demanda devem ser analisadas.

Por hipótese, a curva de oferta do mercado de gasolina C é horizontal. As justificativas para tal hipótese são duas. Em primeiro lugar, o preço da gasolina A é estabelecido considerando aspectos políticos e não só as condições de mercado, o que implica que não há relação bem definida entre preço e quantidade. Em segundo, a quantidade ofertada de álcool anidro é determinada pela quantidade necessária para acrescentar à gasolina A. Mais uma vez, não é possível estabelecer uma relação entre preço e quantidade. Sendo assim, este trabalho assume que a quantidade ofertada de gasolina C, resultado da combinação de $75 \%$ de gasolina A com $25 \%$ de álcool anidro, é estabelecida considerando o preço de mercado. Pelo lado da demanda, por ser a gasolina um bem comum, a inclinação da curva de demanda é negativa.

Diante dessa situação, a introdução do imposto ad valorem ICMS, que por lei incide sobre os produtores, implica um deslocamento da curva de oferta para cima na grandeza exata do tributo, uma vez que é possível interpretá-lo como um aumento dos custos de produção (Gráfico 3.1).

\section{Gráfico 3.1_Efeitos do imposto "ad valorem" sobre as curvas de oferta e demanda do mercado de gasolina C}

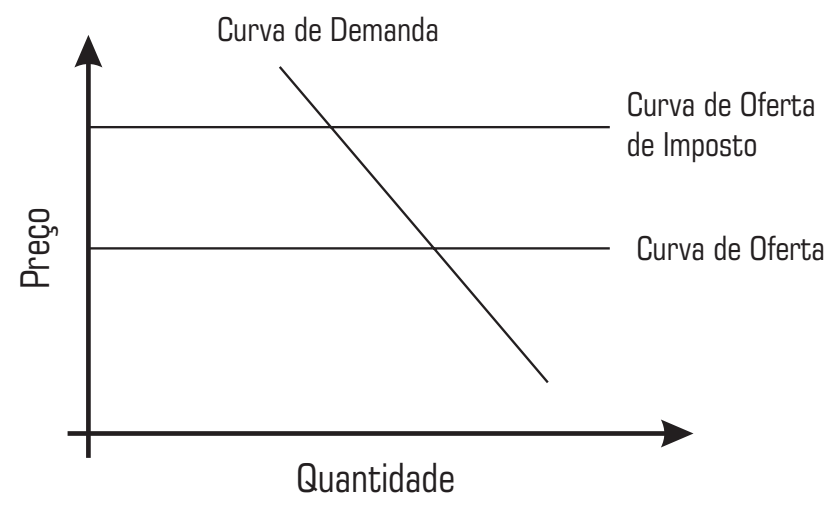

Fonte: Elaboração própria. 
A revisão da literatura é realizada na seção 3.5 , comparando a metodologia e os resultados obtidos nos diversos artigos apresentados com os resultados obtidos neste artigo.
Contudo, sendo a curva de oferta de gasolina perfeitamente elástica, o imposto recai integralmente sobre os consumidores. O preço de oferta de equilíbrio, portanto, permanece inalterado, mas o preço de demanda aumenta exatamente no montante do imposto. Neste caso, o preço de equilíbrio é determinado pelas condições de oferta, enquanto a quantidade de equilíbrio é determinada pela curva de demanda.

Tendo, então, estabelecido as hipóteses sobre a estrutura de mercado, falta determinar as variáveis que, por suposição, afetam a demanda por gasolina C no Brasil. Vale mencionar que as hipóteses são válidas para os sete Estados brasileiros avaliados neste artigo.

Assume-se, portanto, que o consumo de gasolina C é influenciado pelas seguintes variáveis:

i. preço da gasolina C;

ii. preço do álcool hidratado;

iii. renda dos trabalhadores;

iv. frota de automóveis à gasolina;

v. frota de automóveis flex-fuel.

\section{2_ Especificação do modelo matemático}

Considera-se uma relação descrita com base em uma função de demanda condicionada por gasolina C Cobb-Douglas. A adoção dessa função pressupõe que a elasticidade da demanda é constante, mas não necessariamente igual a um. De fato, espera-se que a elasticidade-preço da demanda seja menor que um, caracterizando uma demanda pouco elástica, como encontrado na literatura. ${ }^{1}$

$\mathrm{C}_{\mathrm{g}}=\mathrm{K}\left(\mathrm{P}_{\mathrm{g}}^{\theta_{1}}\right)\left(\mathrm{P}_{\mathrm{a}}^{\theta_{2}}\right)\left(\mathrm{Y}^{\theta_{3}}\right)\left(\mathrm{Fr}_{\mathrm{g}}^{\theta_{4}}\right)\left(\mathrm{Fr}_{\text {flex }}^{\theta_{5}}\right)$

Sendo: $\mathrm{K}=$ constante;

$$
\begin{aligned}
\mathrm{C}_{\mathrm{g}}= & \begin{array}{c}
\text { consumo de gasolina } \mathrm{C} \\
\text { em litro; }
\end{array} \\
\mathrm{P}_{\mathrm{g}}= & \text { preço da gasolina } \mathrm{C} \\
& \text { ( } \$ \text { de } 2008 / \text { litro }) ; \\
\mathrm{P}_{\mathrm{a}}= & \text { preço do álcool hidratado } \\
& \text { (R } \$ \text { de } 2008 / \text { litro); } \\
\mathrm{Y}= & \text { folha de pagamento real } \\
& \text { por trabalhador (índice); } \\
\mathrm{Fr}_{\mathrm{g}}= & \text { frota de automóveis } \\
& \text { a gasolina (unidade); } \\
\mathrm{Fr}_{\text {flex }}= & \text { frota de automóveis } \\
& \text { flex-fuel (unidade). }
\end{aligned}
$$

Vale mencionar que os preços dos combustíveis gasolina e álcool referem-se àqueles praticados pelas distribuidoras e pelos postos revendedores de combustíveis.

Considerando o objetivo deste trabalho de calcular a elasticidade-imposto da demanda por gasolina C, aplicase o logaritmo neperiano à equação (1). 


$$
\begin{aligned}
\ln C_{\mathrm{g}}= & \theta_{\mathrm{o}}+\theta_{1} \ln \mathrm{P}_{\mathrm{g}}+\theta_{2} \ln \mathrm{P}_{\mathrm{a}}+\theta_{3} \ln \mathrm{Y}+ \\
& +\theta_{4} \ln \mathrm{nr}_{\mathrm{g}}+\theta_{5} \ln \mathrm{Fr}_{\text {flex }}, \theta_{0}=\ln \mathrm{K}
\end{aligned}
$$

É possível notar que o imposto estadual ICMS não entra explicitamente na equação. Tendo em vista que o ICMS representa um dos componentes do preço, adicioná-lo ao modelo implicaria contagem dupla. Assim, a fim de verificar o impacto do ICMS no consumo de gasolina C, é preciso aplicar a regra da cadeia.

$\frac{\partial \ln \mathrm{C}_{\mathrm{g}}}{\partial \ln \left(\frac{1}{1-\mathrm{ICMS}}\right)}=\frac{\partial \ln \mathrm{C}_{\mathrm{g}}}{\partial \ln \mathrm{P}_{\mathrm{g}}} \cdot \frac{\partial \ln \mathrm{P}_{\mathrm{g}}}{\partial \ln \left(\frac{1}{1-\mathrm{ICMS}}\right)}$

Por definição, o preço da gasolina C é uma função do preço da gasolina $\mathrm{C}$ sem o ICMS e do próprio imposto estadual.

$\mathrm{P}_{\mathrm{g}}=\frac{\mathrm{P}_{\mathrm{g}}^{\mathrm{s} / \mathrm{ICMS}}}{1-\mathrm{ICMS}}$

Aplicando logaritmo neperiano à equação acima, tem-se que:

$$
\begin{aligned}
\ln P_{g} & =\ln \left(\frac{\mathrm{P}_{g}^{s / I C M S}}{1-\mathrm{ICMS}}\right) \\
& =\ln \mathrm{P}_{\mathrm{g}}^{\mathrm{s} / \mathrm{ICMS}}+\ln \left(\frac{1}{1-\mathrm{ICMS}}\right)
\end{aligned}
$$

Assim, pela equação (5), tem-se que:

$$
\frac{\partial \ln \mathrm{P}_{\mathrm{g}}}{\partial \ln \left(\frac{1}{1-\mathrm{ICMS}}\right)}=1
$$

confirmando a hipótese de repasse integral do imposto sobre a gasolina. Esse resultado indica que calcular a elasticidadeimposto da demanda é o mesmo que calcular a elasticidade-preço da demanda.

De fato, se as elasticidades fossem diferentes, então, os consumidores estariam respondendo de forma diferente aos componentes do preço. Para os consumidores, porém, a variável de interesse é o preço final, sendo, portanto, as razões que resultaram na variação do preço não importantes.

Contudo, cabe mencionar que existe uma possibilidade de a variação no imposto gerar uma variação em algum outro componente do preço, como, por exemplo, as margens de lucro, o que justificaria, nesse caso, encontrar uma elasticidade-imposto diferente da elasticidade-preço da demanda. Medir isso, entretanto, requereria a formulação de uma função de mark-up dos revendedores de gasolina C. 


\section{3_ Especificação do modelo econométrico}

O modelo matemático pressupõe uma relação exata entre as variáveis; contudo, essa relação não se verifica na prática. A demanda por gasolina C é provavelmente influenciada por outras variáveis não explicitadas no modelo, como, por exemplo, os hábitos dos consumidores. Sendo assim, acrescentamos ao modelo determinístico, descrito anteriormente, um termo denominado "distúrbio", w.

$$
\begin{aligned}
\ln \mathrm{C}_{\mathrm{g}}= & \theta_{\mathrm{o}}+\theta_{1} \ln \mathrm{P}_{\mathrm{g}}+\theta_{2} \ln \mathrm{P}_{\mathrm{a}}+\theta_{3} \ln \mathrm{Y}+(6) \\
& +\theta_{4} \ln \mathrm{Fr}_{\mathrm{g}}+\theta_{5} \ln \mathrm{Fr}_{\text {flex }}+\mathrm{w}
\end{aligned}
$$

As propriedades do distúrbio são conhecidas, sendo w um ruído branco. Ou seja, o termo de erro aleatório é não correlacionado com as variáveis explicativas, com média zero e variância constante.

Além disso, com base na observação dos dados referentes à demanda por gasolina $\mathrm{C}$, notou-se um comportamento sazonal. Em geral, no final do ano há um aumento do consumo de gasolina $\mathrm{C}$ motivado, provavelmente, pelo aumento da renda, $13^{\circ}$ salário, e pelas férias e festas do fim do ano. Em contrapartida, no início do ano, há forte queda do consumo, resultado, talvez, da diminuição de renda graças à grande quantidade de impostos a serem pagos nessa época. A fim de captar tais movimentos, 11 dum- mies sazonais foram adicionadas ao modelo, sendo janeiro considerado, aleatoriamente, o mês-base.

$$
\begin{aligned}
\ln C_{\mathrm{g}}= & \theta_{\mathrm{o}}+\theta_{1} \ln \mathrm{P}_{\mathrm{g}}+\theta_{2} \ln \mathrm{P}_{\mathrm{a}}+\theta_{3} \ln \mathrm{Y}+\quad(7) \\
& +\theta_{4} \ln \mathrm{Fr}_{\mathrm{g}}+\theta_{5} \ln \mathrm{Fr}_{\text {flex }}+\theta_{6} \mathrm{Fev}+ \\
& +\theta_{7} \mathrm{Mar}+\theta_{8} \mathrm{Abr}+\theta_{9} \mathrm{Mai}+\theta_{10} \text { Jun }+ \\
& +\theta_{11} \mathrm{Jul}+\theta_{12} \mathrm{Ago}+\theta_{13} \mathrm{Set}+ \\
& +\theta_{14} \text { Out }+\theta_{15} \mathrm{Nov}+\theta_{16} \mathrm{Dez}+\mathrm{w}
\end{aligned}
$$

Cabe acrescentar que, inicialmente, se tentou controlar para mudanças nos impostos federais, introduzindo-os ao modelo. Contudo, de forma simétrica, ao imposto estadual, ICMS, os impostos federais, CIDE, PIS/PASEP e COFINS, são componentes do preço final da gasolina $\mathrm{C}$ e adicioná-los ao modelo implicaria multicolinearidade perfeita e, por isso, foram retiradas do modelo.

\section{4_ Obtenção dos dados}

Como mencionado anteriormente, a estimação do modelo econométrico é realizada para sete Estados brasileiros em razão da falta de disponibilidade de dados para todos os Estados mais o Distrito Federal. Além disso, o período de análise é mensal, de julho de 2001 a dezembro de 2008.

Como não há dados sobre consumo de gasolina $\mathrm{C}$, foram utilizadas as 
vendas desse combustível pelas distribuidoras, disponibilizadas pela Agência Nacional de Petróleo, Gás e Biocombustíveis. Cabe mencionar que os dados referentes à venda têm como unidade de medida o metro cúbico, mas, como os preços tanto da gasolina $\mathrm{C}$ quanto do álcool hidratado estão em real por litro, as vendas foram multiplicadas por mil para que ficassem na mesma unidade de volume dos preços.

As informações sobre os preços dos combustíveis também são disponibilizadas pela Agência Nacional de Petróleo. Dado o interesse de trabalhar com valores reais, foi necessário deflacionar os preços. Para isso, utilizou-se o Índice Geral de Preços (IGP-M), divulgado pela Fundação Getúlio Vargas. A base original do índice é agosto de 1994; entretanto, mudou-se a base para dezembro de 2008.

Em relação à renda, foi utilizada a Folha de Pagamento Real por Trabalhador, divulgada pelo IBGE, relativa à indústria geral. A Folha de Pagamento é, na verdade, um índice de base fixa, janeiro de 2001, sem ajuste sazonal. De forma análoga ao IGP-M, mudou-se a base para dezembro de 2008. Esse índice não é divulgado para todos os Estados brasileiros de forma que, para os do Mato Gros- so do Sul e de Sergipe, foram utilizados os dados referentes às regiões CentroOeste e Nordeste, respectivamente.

Os dados referentes à frota de automóveis, seja esses a gasolina, seja esses flex-fuel, foram obtidos no Detran dos sete Estados já mencionados. Com exceção do Rio de Janeiro e de Sergipe, os dados disponibilizados são anuais, de forma que foi preciso transformar esses em mensais. Para isso, a taxa anual de crescimento foi calculada, e, com base nessa, a taxa de crescimento mensal.

Considerando, portanto, o modelo e os dados, a próxima etapa é calcular os parâmetros do modelo especificado.

\section{5_ Estimação dos parâmetros do modelo econométrico}

O modelo econométrico (7) é estimado com base na econometria de séries temporais. A avaliação, portanto, é realizada utilizando-se o software Eviews 4.1 para os sete Estados de forma independente.

Para estimar a elasticidade-preço da demanda por gasolina C, os parâmetros foram calculados via mínimos quadrados ordinários. Já, refletindo diferentes comportamentos quanto ao consumo de gasolina C, o modelo (7) teve de ser adaptado para alguns Estados. 
Para Pernambuco, Paraná e Mato-Grosso do Sul, o modelo original (7) foi utilizado. A técnica de cointegração e o mecanismo de correção de erro foram aplicados a fim de estimar as elasticidades-preço da demanda de longo e de curto prazos, respectivamente. Além disso, as variáveis consideradas na regressão, de acordo com o teste de Hausman, são exógenas, com exceção da própria variável dependente "Consumo de Gasolina C".

Com relação ao Ceará e a Sergipe, as variáveis "Preço Real do Álcool Hidratado", "Frota de Automóveis a Gasolina e Flex-Fuel" foram consideradas endógenas. Desta forma, especificou-se um novo modelo e também se rodou um VAR bivariado, sendo as variáveis "Preço Real da Gasolina C" e "Renda Real por Trabalhador" exógenas, enquanto as variáveis "Consumo de Gasolina C" e "Preço Real do Álcool Hidratado", endógenas. Repare que as séries referentes às frotas foram excluídas, já que essas, em quaisquer modelos, eram sempre estatisticamente não significativas.

Entretanto, mesmo incluindo a defasagem das variáveis endógenas até a terceira ordem, não foi possível eliminar a correlação serial de segunda e terceira ordem do modelo VAR bivariado, de forma

que o seguinte modelo foi estimado tanto para o Ceará quanto para Sergipe.

$$
\begin{aligned}
\ln C_{\mathrm{gt}}= & \theta_{0}+\theta_{1} \operatorname{lnC}_{\mathrm{g}_{\mathrm{t}-1}}+\theta_{2} \ln \mathrm{C}_{\mathrm{g}_{\mathrm{t}-12}}+ \\
& +\theta_{3} \ln _{\mathrm{g}_{\mathrm{t}-13}}+\theta_{4} \ln \mathrm{P}_{\mathrm{gt}}+\theta_{5} \ln \mathrm{Y}_{\mathrm{t}}+ \\
& +\theta_{6} \ln \mathrm{Y}_{\mathrm{t}-1}+\theta_{7} \ln \mathrm{Y}_{\mathrm{t}-12}+\theta_{8} \ln \mathrm{Y}_{\mathrm{t}-13}+ \\
& +\theta_{9} \mathrm{Fev}+\theta_{10} \mathrm{Mar}+\theta_{11} \mathrm{Abr}+ \\
& +\theta_{12} \mathrm{Mai}+\theta_{13} \mathrm{Jun}+\theta_{14} \mathrm{jul}+ \\
& +\theta_{15} \text { Ago }+\theta_{16} \operatorname{Set}+\theta_{17} \text { Out }+ \\
& +\theta_{18} \mathrm{Nov}+\theta_{19} \text { Dez }+\tau_{20} \mathrm{t}+\mathrm{w}_{\mathrm{t}}
\end{aligned}
$$

A escolha das defasagens foi baseada na análise da função de correlação parcial. Já nos Estados do Rio de Janeiro e do Rio Grande do Sul, apesar de todos os regressores serem exógenos, foi preciso especificar um novo modelo. No caso do Rio de Janeiro, especificou-se uma regressão adicional, considerando o preço do gás natural veicular, visto que a frota de automóveis a gás no Rio é alta.

Deste modo, as equações estimadas para o Rio de Janeiro, cujos resultados são apresentados abaixo, são:

$$
\begin{aligned}
\ln C_{\mathrm{gt}} & =\theta_{0}+\theta_{1} \ln \mathrm{P}_{\mathrm{gt}}+\theta_{2} \ln \mathrm{P}_{\mathrm{at}}+ \\
& +\theta_{3} \ln \mathrm{Y}_{\mathrm{t}}+\theta_{4}\left(\operatorname{lnFr}_{\mathrm{gt}}^{2}\right)+\theta_{5}\left(\operatorname{lnFr}_{\text {flext }}^{2}\right)+ \\
& +\theta_{6} \mathrm{Fev}+\theta_{7} \mathrm{Mar}+\theta_{8} \mathrm{Abr}+\theta_{9} \mathrm{Mai}+ \\
& +\theta_{10} \mathrm{Jun}+\theta_{11} \mathrm{Jul}+\theta_{12} \text { Ago }+ \\
& +\theta_{13} \mathrm{Set}+\theta_{14} \mathrm{Out}+\theta_{15} \mathrm{Nov}+ \\
& +\theta_{16} \mathrm{Dez}+\theta_{17} \mathrm{t}+\mathrm{w}_{\mathrm{t}}
\end{aligned}
$$




$$
\begin{aligned}
\ln C_{\mathrm{gt}}= & \theta_{0}+\theta_{1} \ln \mathrm{P}_{\mathrm{gt}}+\theta_{2} \ln \mathrm{P}_{\mathrm{gt}}+ \\
& +\theta_{3} \ln \mathrm{Y}_{\mathrm{t}}+\theta_{4}\left(\operatorname{lnFr}_{\mathrm{gt}}^{2}\right)+\theta_{5}\left(\operatorname{lnFr}_{\text {flext }}^{2}\right)+ \\
& +\theta_{6} \mathrm{Fev}+\theta_{7} \mathrm{Mar}+\theta_{8} \mathrm{Abr}+\theta_{9} \mathrm{Mai}+ \\
& +\theta_{10} \mathrm{Jun}+\theta_{11} \mathrm{Jul}+\theta_{12} \text { Ago }+ \\
& +\theta_{13} \text { Set }+\theta_{14} \text { Out }+\theta_{15} \mathrm{Nov}+ \\
& +\theta_{16} \mathrm{Dez}+\theta_{17} \mathrm{t}+\mathrm{w}_{\mathrm{t}}
\end{aligned}
$$

No caso do Rio Grande do Sul, o modelo econométrico utilizado foi basicamente a regressão (7) sem as séries referentes à frota de automóveis a gasolina e à frota flex-fuel.

$$
\begin{aligned}
\ln C_{g t}= & \theta_{0}+\theta_{1} \ln P_{g t}+\theta_{2} \ln P_{a t}+ \\
& +\theta_{3} \ln Y_{\mathrm{t}}+\theta_{4} \mathrm{Fev}+\theta_{5} \text { Mar }+ \\
& +\theta_{6} \mathrm{Abr}+\theta_{7} \mathrm{Mai}+\theta_{8} \text { Jun }+ \\
& +\theta_{9} \mathrm{Jul}+\theta_{10} \text { Ago }+\theta_{11} \text { Set }+ \\
& +\theta_{12} \text { Out }+\theta_{13} \text { Nov }+ \\
& +\theta_{14} \text { Dez }+\theta_{15} \mathrm{t}+\mathrm{w}_{\mathrm{t}}
\end{aligned}
$$

Assim sendo, os resultados obtidos são apresentados na Tabela 3.1.

Com exceção de Pernambuco, as elasticidades-preço da demanda por gasolina $\mathrm{C}$ tanto de curto quanto de longo prazo são baixas, o que está de acordo com os resultados obtidos na literatura.

Para Burnquist e Bacchi (2002), que avaliaram o comportamento da demanda por gasolina, entre 1973 e 1998, com base em dados anuais, os resultados obtidos sugerem uma baixa elasticidadepreço da demanda tanto de curto quanto de longo prazo, $-0,319$ e $-0,227$, respectivamente. Considerando basicamente o mesmo período, 1974 a 1999, e a mesma técnica econométrica de cointegração e o mecanismo de correção de erro, Alves e Bueno (2003) encontram uma inelasticidade de curto prazo e uma baixa elasticidade de longo prazo, $-0,464$.

As elasticidades-preço da demanda de longo prazo calculadas pelos autores Pinto et al. (2006), Nappo (2007) e Schünemann (2007) foram também baixas, $-0.141,-0.196$ e -0.3176 , respectivamente; contudo, é preciso fazer algumas ressalvas. Primeiro, apesar de os três trabalhos utilizarem dados mensais, o período de análise difere entre eles. No caso de Pinto et al. (2006), os dados correspondem ao período de julho de 2001 a agosto de 2006. Já, no caso de Nappo (2007), ao de agosto de 1994 a julho de 2006 e, finalmente, no que se refere a Schünemann (2007), de julho de 2001 a fevereiro de 2007. Segundo, Pinto et al. (2006) utilizam dados em painéis para estimar a elasticidade-preço da demanda por gasolina no Brasil, enquanto os demais autores usam a técnica de cointegração e o mecanismo de correção de erro. 
Tabela 3.1_ Resultado das estimações: elasticidade-preço da demanda por gasolina C nos sete Estados brasileiros

\begin{tabular}{|c|c|c|c|c|}
\hline \multirow[b]{2}{*}{ Unidades da Federação } & \multicolumn{3}{|c|}{ Curto prazo } & \multirow{2}{*}{$\begin{array}{l}\text { Longo prazo } \\
\text { Coeficiente }\end{array}$} \\
\hline & $\begin{array}{c}\text { Coefciente } \\
\text { (Desvio-Padrão) }\end{array}$ & Estatística & P-Valor & \\
\hline Ceará & $\begin{array}{l}-0.207934 \\
(0.071802) \\
\end{array}$ & -2.895944 & 0.0052 & -0.14658 \\
\hline Pernambuco & $\begin{array}{l}-0.707052 \\
(0.218612)\end{array}$ & -3.234274 & 0.0024 & -0.68787 \\
\hline Sergipe & $\begin{array}{l}-0.145449 \\
(0.093861)\end{array}$ & -1.549630 & 0.1264 & -0.10270 \\
\hline $\begin{array}{l}\text { Rio de Janeiro } \\
\text { (equação 9) }\end{array}$ & $\begin{array}{l}-0.218402 \\
(0.421429) \\
\end{array}$ & -0.518242 & 0.6071 & -0.59222 \\
\hline $\begin{array}{l}\text { Rio de Janeiro } \\
\text { (equação 10) }\end{array}$ & $\begin{array}{l}-0.074312 \\
(0.172895)\end{array}$ & -0.429808 & 0.6688 & -0.06023 \\
\hline Paraná & $\begin{array}{c}0.050930 \\
(0.269595)\end{array}$ & 0.188912 & 0.8514 & -0.17940 \\
\hline Rio Grande do Sul & $\begin{array}{l}-0.577913 \\
(0.183386)\end{array}$ & -3.151352 & 0.0024 & -0.58929 \\
\hline Mato Grosso do Sul & $\begin{array}{l}-0.356696 \\
(0.427716)\end{array}$ & -0.833957 & 0.4166 & -0.38300 \\
\hline
\end{tabular}

Fonte: Elaboração própria.

Finalmente, o trabalho recente de Miootty et al. (2009) sobre consumo de combustíveis automotivos entre 1970 e 2005 estima as elasticidades-preço e renda via uma aproximação linear utilizando o modelo Almost Ideal Demand System. Diferentemente dos demais trabalhos apresentados, os resultados obtidos em Miootty et al. (2009) sugerem alta elasticidade-preço da demanda por gasolina,
$-3,84 \%$. Segundo os autores, esse resultado pode ser considerado como uma evidência da alta substubilidade entre a gasolina e o álcool hidratado.

De qualquer forma, vale ressaltar que os estudos explicitados acima são referentes ao Brasil, sendo difícil, portanto, qualquer comparação direta. A importância dos resultados obtidos neste artigo encontra-se nas diferentes respostas entre 
os Estados brasileiros quanto às mudanças no preço da gasolina C. Entretanto, deve-se ter atenção, já que nem todos os parâmetros estimados são significativos.

A não significância estatística dos testes $\mathrm{t}$ individuais, os altos valores encontrados de $\mathrm{R}^{2}$, superiores a 0,8 , e a significância dos testes $\mathrm{F}$ de significância conjunta podem indicar a presença de multicolinearidade entre os regressores. A princípio, pouco pode ser feito, uma vez que a multicolinearidade é essencialmente um problema de deficiência de dados (Gujarati, 2006). Contudo, é preciso considerar que o problema da multicolinearidade está associado a seu grau, e não a sua existência.

Com base na matriz de correlações, existe alta correlação entre as séries "Preço Real da Gasolina C" e "Preço Real do Álcool Hidratado" e também alta correlação entre as séries "Frota de Automóveis à Gasolina" e "Frota de Automóveis Flex-Fuel'. Cabe acrescentar, entretanto, que, num modelo com mais de duas variáveis explanatórias, como é o caso, a correlação simples, ou de ordem zero, não corresponde a um indicador exato de presença de multicolinearidade e, portanto, não é possível definir uma relação direta entre alta correlação simples e multicolinearidade.
Outro fator que pode estar ocasionando a não significância individual dos regressores é a possível existência de erro de especificação. Contudo, o teste RESET de Ramsey foi realizado, e todos os modelos apresentados foram corretamente especificados de acordo com esse teste. Cabe mencionar que o teste de Chow não foi feito em razão do tamanho pequeno da amostra. Logo, pouco pode ser feito, sendo a deficiência de dados a causa mais plausível para explicar a não significância estatística dos testes $t$ individuais.

Descobrir o porquê das diferenças é importante a fim de considerar as especificidades de cada Estado na formulação de políticas públicas. A princípio, as diferentes alíquotas entre os Estados podem ser responsáveis pelas distintas respostas, mas, considerando que Ceará e Pernambuco têm as mesmas alíquotas de ICMS sobre a gasolina, essas não representam a única razão.

Os demais componentes dos preços finais de gasolina $\mathrm{C}$ aos consumidores, como, por exemplo, o frete e as margens, também podem ser apontados como fatores responsáveis pelo diferente comportamento dos consumidores. Com base na análise dos preços reais da gasolina C, é possível notar que os preços 
reais no Paraná, em geral, são menores que nos outros Estados, o que explicaria a baixa elasticidade-preço da demanda por gasolina C, uma vez que os incentivos relativos ao custo da gasolina são menores nesse Estado, não havendo, assim, estímulo a mudar de combustível.

É válido frisar que esse tipo de análise é puramente econômica, não considerando, portanto, questões relativas à preocupação ambiental que os consumidores possam vir a ter e, nesse sentido, serem estimulados a escolher combustíveis menos poluentes.
É possível argumentar também que talvez o importante para o consumidor seja o preço relativo, e não o preço absoluto da gasolina C. Nesse caso, sendo o álcool hidratado o substituto mais próximo e utilizando a regra de equivalência dos combustíveis, tem-se que os consumidores paranaenses deveriam consumir apenas álcool hidratado, uma vez que, com exceção do primeiro semestre de 2006, o preço do álcool esteve sempre abaixo dos $70 \%$ do preço da gasolina C (Gráfico 3.2).

\section{Gráfico 3.2_Razão entre o preço real do álcool hidratado e o preço real da gasolina C para os sete Estados brasileiros}

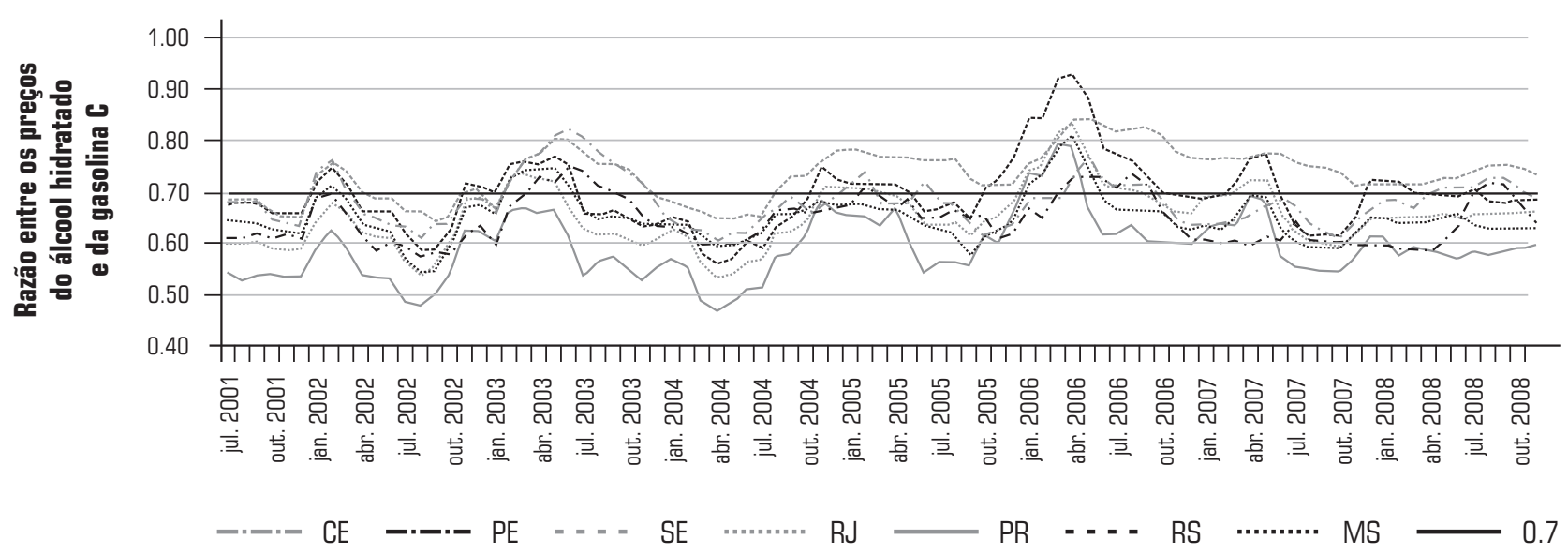

Fonte: ANP (2009) e Ipeadata (2009). 
Contudo, como já visto, o Estado do Paraná é o que apresenta a menor elasticidade, de forma que outros fatores, que não econômicos, têm peso maior na decisão dos consumidores. Talvez seja o simples desconhecimento quanto a essa regra ou talvez sejam as questões comportamentais responsáveis pelas diferentes respostas entre os Estados.

\section{4_Conclusão}

A análise de sensibilidade do mercado de gasolina $\mathrm{C}$ nos sete Estados brasileiros avaliados mostrou que existem diferenças significativas entre esses. As reações a mudanças no preço final da gasolina $\mathrm{C}$ divergem consideravelmente.

Cabe mencionar que a indisponibilidade de dados para alguns Estados impossibilitou a análise econométrica das reações do consumo a mudanças no preço via variações nas alíquotas do imposto estadual ICMS sobre a gasolina para todas as Unidades da Federação, entre elas São Paulo, maior consumidor brasileiro de gasolina C. Acredita-se, porém, que o estudo permanece válido à medida que o tema "viabilidade da política tributária como ferramenta de política ambiental e energética" é válido para todos os Estados. Assim sendo, analisou-se, neste artigo, sete Estados - Ceará, Pernambuco, Sergipe, Rio de Janeiro, Paraná, Rio Grande do Sul e Mato Grosso do Sul. Considerando apenas aqueles cujas respostas foram significativas, tem-se que, no Rio Grande do Sul e, principalmente, em Pernambuco, políticas de preço poderiam ser utilizadas como instrumentos de políticas energéticas e ambientais.

Assim, tendo como perspectiva o desenvolvimento econômico sustentável, o ICMS poderia ser utilizado para reduzir o consumo de gasolina $\mathrm{C}$ em alguns Estados específicos. De fato, o potencial desse imposto estadual pôde ser observado em 4 de dezembro de 2003, em São Paulo, que reduziu o ICMS sobre o álcool hidratado de $25 \%$ para $12 \%$. Consequentemente, $80 \%$ dos donos de automóveis flex-fuel abastecem com álcool.

O uso do ICMS como política energética e ambiental oferece expressiva vantagem em relação às demais políticas públicas. Sendo o Brasil um país muito grande, os Estados apresentam diversas particularidades que, em geral, não são consideradas na formulação de políticas nacionais.

A fim de comparar as respostas dos Estados avaliados com a reação nacional a mudanças no ICMS sobre a gaso- 
lina, estimou-se o mesmo modelo econométrico (5), mas, para a estimação desse modelo, foram utilizados dados em painel, em que i representa os sete Estados avaliados, e t, o mês.

$$
\begin{aligned}
\operatorname{lnC}_{\mathrm{g}_{\mathrm{it}}}= & \theta_{0}+\theta_{1} \ln \mathrm{P}_{\mathrm{g}_{\mathrm{it}}}+\theta_{2} \operatorname{lnP}_{\mathrm{a}_{\mathrm{it}}}+ \\
& +\theta_{3} \ln \mathrm{Y}_{\mathrm{it}}+\theta_{4} \operatorname{lnFr}_{\mathrm{g}_{\mathrm{it}}}+\theta_{5} \ln \mathrm{Fr}_{\mathrm{flex}_{\mathrm{it}}}+ \\
& +\theta_{6} \mathrm{Fev}+\theta_{7} \mathrm{Mar}+\theta_{8} \mathrm{Abr}+ \\
& +\theta_{9} \mathrm{Mai}+\theta_{10} \mathrm{Jun}+\theta_{11} \mathrm{Jul}+ \\
& +\theta_{12} \text { Ago }+\theta_{13} \mathrm{Set}+\theta_{14} \mathrm{Out}+ \\
& +\theta_{15} \mathrm{Nov}+\theta_{16} \text { Dez }+\mathrm{w}_{\mathrm{it}}
\end{aligned}
$$

Onde $i=1, \ldots, 7$ e $t=$ jan01, ..., dez08

O modelo econométrico especificado acima procurou representar a realidade do mercado de gasolina $\mathrm{C}$ no Brasil, ainda que seja praticamente impossível uma representação perfeita.

Além disso, assumiu-se certa inércia no comportamento do consumo de gasolina $\mathrm{C}$, de forma que a variável de- pendente foi defasada uma vez e introduzida no modelo como variável predeterminada. A estimação dos parâmetros foi realizada via Arellano e Bond (1991). Os resultados de interesse são apresentados na Tabela 4.1.

Valendo-se dos resultados encontrados, é possível notar uma elasticidadepreço da demanda por gasolina $\mathrm{C}$ baixa, mas não inelástica.

Entretanto, o ponto que deve ser destacado é a diferença da resposta nacional em relação às respostas estaduais. Desta forma, estratégias baseadas no uso de políticas públicas nacionais que não consideram as especificidades de cada Estado provavelmente não serão eficazes ou terão eficácia menor. Isso porque

\begin{tabular}{|c|c|c|c|c|}
\hline & $\begin{array}{c}\text { Coeficiente } \\
\text { (Desvio-Padrão) }\end{array}$ & Estatística de Testes & P-Valor & $\begin{array}{c}\text { Intervalo de } \\
\text { Confiança }{ }^{\text {(a) }} \\
(\alpha=10 \%)\end{array}$ \\
\hline Curto prazo & $\begin{array}{r}-0.5147584 \\
(0.073604) \\
\end{array}$ & -6.99 & 0.000 & $(-0.6358,-0.3936)$ \\
\hline Longo prazo & -0.4796999 & - & - & - \\
\hline
\end{tabular}
um aumento de $1 \%$ no preço da gasolina C implicaria, no curto prazo, redução de $0,51 \%$, enquanto, por exemplo, no Ceará, a queda seria de apenas $0,20 \%$.

Tabela 4.1_ Painel nacional: elasticidades-preço da demanda por gasolina C de curto e longo prazos

(a) $\operatorname{Pr}\left[\hat{\beta}-t_{\alpha / 2} \operatorname{dp}(\hat{\beta}) \leq \beta \leq \hat{\beta}+t_{\alpha / 2} \operatorname{dp}(\hat{\beta})\right]=1-\alpha$

Fonte: Elaboração própria. 
Posto isso, é grande a relevância de políticas públicas estaduais nos objetivos do governo federal, uma vez que essas aumentam a probabilidade de que os recursos financeiros públicos sejam alocados de maneira mais eficiente e eficaz.

Vale ressaltar que o objetivo deste trabalho foi o de avaliar a viabilidade da política tributária estadual como ferramenta de políticas ambientais e energéticas, ficando para um próximo trabalho uma discussão mais aprofundada sobre os impactos da adoção do ICMS como instrumento de política pública no orçamento. De qualquer forma, cabe aqui destacar um breve comentário sobre esse ponto.

Até então, não foi discutido o impacto do uso de políticas tributárias, com outro fim que não o da arrecadação, no orçamento dos Estados brasileiros. O ICMS representa, para muitos deles, parte significativa do orçamento, e, por isso, tal discussão deve ser considerada neste trabalho. Em São Paulo, por exemplo, principal consumidor de gasolina $\mathrm{C}$, a participação relativa do ICMS, em 2009, foi de $34,4 \%$, de acordo com a Comissão Técnica Permanente do ICMS (Cotepe), e a arrecadação específica com petróleo, combustíveis e lubrificantes representou, em 2009, 10,18\% da arrecadação to- tal do ICMS. Sendo assim, é notório que o ICMS é uma das principais fontes de arrecadação, e a utilização desse imposto sobre a gasolina C, a fim de reduzir o consumo dessa, pode gerar perda de arrecadação para os Estados, de forma que resistências a implementação de tais políticas podem surgir. Contudo, cálculos mais precisos são necessários com o intuito de verificar se esse tipo de política tributária realmente se traduziria em diminuição do orçamento dos Estados.

Dependendo do aumento do ICMS e da queda do consumo do combustível, a arrecadação pode até nem ser alterada. Outro cálculo necessário seria o ganho de arrecadação em decorrência do aumento do consumo de combustíveis alternativos, como, por exemplo, o álcool. Em São Paulo, a compensação seria mais difícil visto que o valor da alíquota do ICMS sobre o álcool é de $12 \%$. Todavia, nos Estados encontrados neste artigo como possíveis de aplicarem a política tributária como instrumento de política energética e ambiental, nos do Ceará, de Pernambuco e do Rio Grande do Sul, a compensação talvez seja viável dado que a alíquota do ICMS sobre o álcool é igual a $25 \%$. 


\section{Referências bibliográficas}

ALVES, D. C. O.; BUENO, R. D. L. S. Short-run, long-run and cross elasticities of gasoline demand in Brazil. Energy Economics, n. 25, p. 191-199, 2003.

ARELLANO, M.; BOND, S. R. Some tests of specification for panel data: Monte Carlo evidence and an application to employment equations. Review of Economic Studies, v. 58 , p. 277-297, 1991.

\section{ANFAVEA. ASSOCIAÇÃO}

NACIONAL DOS

FABRICANTES DE VEÍCULOS AUTOMOTORES. Disponível em: <http://www.anfavea.com. br>. Acesso em: 23 ago. 2009.

ANP. AGÊNCIA NACIONAL DE PETRÓLEO,

GÁS NATURAL E

BIOCOMBUSTÍVEIS. Disponível em: <http://www.anp.gov.br>. Acesso em: 23 abr. 2009.

BEN. Balanço Energético Nacional. Empresa de Pesquisa Energética. Rio de Janeiro: EPE, 2008. Disponível em: <http:// www.epe.gov.br $>$. Acesso em: 17 abr. 2009.
BURNQUIST, H. L.; BACCHI,

M. R. P. A demanda por gasolina no Brasil: uma análise utilizando técnicas de co-integração. In: CONGRESSO BRASILEIRO DE ECONOMIA E SOCIOLOGIA RURAL, 40., 2002, Passo Fundo. Anais... Passo Fundo: EDIUPF, 2002.

\section{COTEPE. CONSELHO}

TÉCNICO PERMANENTE DO ICMS. Disponível em: <http:// www.fazenda.gov.br/confaz/ boletim/>. Acesso em:

23 abr. 2009.

DENATRAN. DEPARTAMENTO NACIONAL DE TRÂNSITO.

Disponível em: <http://www. denatran.gov.br $>$. Acesso em: 23 abr. 2009.

DETRAN/CE. DEPARTAMENTO DE TRÂNSITO DO CEARÁ. Disponível em: <http://www. detran.ce.gov.br $>$. Acesso em: 23 de abril de 2009 .

\section{DETRAN/MS}

DEPARTAMENTO DE

TRÂNSITO DO MATO GROSSO DO SUL. Disponível em: <http://www.detran.ms.gov. br>. Acesso em:23 abr. 2009.
DETRAN/PE.

DEPARTAMENTO

DE TRÂNSITO DO

PERNAMBUCO. Disponível em: $<$ http://www.detran.pe.gov.br>. Acesso em: 23 abr. 2009.

DETRAN/PR.

DEPARTAMENTO DE TRÂNSITO DO PARANÁ.

Disponível em: $<$ http://www. detran.pr.gov.br $>$. Acesso em: 23 abr. 2009.

DETRAN/RJ.

DEPARTAMENTO DE

TRÂNSITO DO RIO DE

JANEIRO. Disponível em:

$<$ http://www.detran.ri.gov.br>.

Acesso em: 23 abr. 2009.

DETRAN/RS.

DEPARTAMENTO DE

TRÂNSITO DO RIO GRANDE

DO SUL. Disponível em: <http://

www.detran.rs.gov.br>.

Acesso em: 23 abr. 2009.

DETRAN/SE.

DEPARTAMENTO DE TRÂNSITO DO SERGIPE.

Disponível em: <http://www. detran.se.gov.br>. Acesso em: 23 abr. 2009.

GUJARATI, D. N.

Econometria básica. Rio de Janeiro: Elsevier, 2006.

( $3^{\mathrm{a}}$ reimpressão).
IBGE. INSTITUTO

BRASILEIRO DE GEOGRAFIA E ESTATÍSTICA. Disponível em: <http://www.ibge.gov.br>. Acesso em: 20 ago. 2009.

IBP. INSTITUTO BRASILEIRO DE PETRÓLEO, GÁS E BIOCOMBUSTÍVEIS. Vale Apena Usar o GNV. GNV News. Rio de Janeiro, v. 3, n. 38, p. 1, jul. 2008. Disponível em: <http:// www.ibp.org.br/services/.../ FileDownload.EZTSvc.asp?>. Acesso em: 30 abr. 2009.

IPEADATA. INSTITUTO DE PESQUISA ECONÔMICA APLICADA. Disponível em: <http://www.ipeadata.gov.br> Acesso em: 30 abr. 2009.

MIOOTTY et al. Automotive fuel consumption in Brazil: Applying static and dynamic systems of demand equations. Energy Policy, v. 37, p. 5326-5333, 2009.

NAPPO, M. A. Demanda por gasolina no Brasil: uma avaliação de suas elasticidades após a introdução dos carros bicombustível. 2007. $61 \mathrm{f}$. Dissertação (Mestrado em Finanças e Economia Industrial) Fundação Getúlio Vargas, Escola de Economia de São Paulo, São Paulo, 2007. 
NOTA TÉCNICA ANP

013/2001. Combustíveis no Brasil: políticas de preço e estrutura tributária, 2001.

PINTO JR. et al. Matriæ Brasileira de Combustiveis. Grupo de

Economia de Energia - Instituto de Economia/UFRJ e Centro de Gestão de Estudos Estratégicos Núcleo de Assuntos Estratégicos da Presidência da República, 2006. Disponível em: <http://www.nae. gov.br/cadernos_nae/10relatorio_ final_matriz_de_combustivel. pdf>. Acesso em: 20 ago. 2009.

SCHÜNEMANN, L. $A$ demanda de gasolina automotiva no Brasil: $\mathrm{o}$ impacto nas elasticidades de curto e longo prazo da expansão do GNV e dos carros Flex. 2007. 91 f. Dissertação (Mestrado profissionalizante em Economia) Faculdade de Economia e Finanças do IBMEC, Rio de Janeiro, 2007.

SINDICON. SINDICATO

NACIONAL DAS EMPRESAS

DISTRIBUIDORAS DE

COMBUSTÍVEIS E DE

LUBRIFICANTES - Mapa do ICMS. 2008. Disponível em:

$<$ http://www.sindicom.com.br>. Acesso em: 30 abr. 2009.

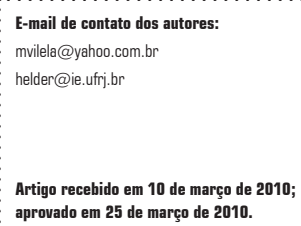

